\title{
La percepción de los alumnos de Secundaria y Bachillerato hacia la inclusión de compañeros con discapacidad en Educación Física
}

\author{
Carmen OCETE ${ }^{1}$,, Carmen LAMATA ${ }^{1}$, Javier COTERÓN², Luis J. DURÁN², \\ y Javier PÉREZ-TEJERO'. \\ ${ }^{1}$ Cátedra de Estudios sobre el Deporte Inclusivo. Universidad Politécnica de Madrid. \\ ${ }^{2}$ Universidad Politécnica de Madrid.
}

(Recibido 30 Marzo, 2017; Aceptado 18 Mayo, 2017)

RESUMEN: El objetivo de este estudio fue conocer la percepción de los alumnos sin discapacidad sobre la inclusión de compañeros que sí la tienen en las clases de Educación Física (EF) a través del análisis de dibujos y frases expresadas durante la actividad de reflexión en el evento deportivo inclusivo "Deporte Inclusivo en la Escuela". En esta actividad los alumnos reflejaron sus opiniones, sensaciones y vivencias sobre la inclusión de compañeros con discapacidad en las sesiones de EF. El análisis de las frases y las palabras $(n=296)$ se basó en la "Teoría de la estructura y el contenido universal de los valores humanos" (Schwartz, 1992), mientras que los dibujos ( $\mathrm{n}=50)$ fueron analizados desde la "Teoría de la Retórica visual" (Barthes, 1964), ya que el dibujo se convierte en una herramienta para conocer cómo perciben los alumnos la inclusión en EF. Los resultados de las frases muestran que las percepciones de los alumnos tienden hacía dominios motivacionales colectivistas y mixtos, como universalismo y logro, coincidiendo con los mostrados por los resultados de los dibujos. Otros valores relacionados con la inclusión emergen a su vez, como autodirección y benevolencia.

Palabras clave: inclusión, percepción, discapacidad, Educación Física, deporte inclusivo.

\section{High school students's perception towards inclusion of peers with disability in Physical Education}

\begin{abstract}
The aim of this study was to know the perception of students without disabilities toward the inclusion of peers with disability Physical Education (PE) class, through the analysis of the images and phrases expressed during the reflection activity in the inclusive sport event "Inclusive Sport in School". In this activity, students reflect their opinions, feelings and experiences through phrases, words and drawings. The analysis of the sentences, words $(n=296)$ was carried out using a system of categories based on Schwartz's "Theory of the structure and universal content of human values" (Schwartz, 1992), while the images $(n=50)$ were analyzed from the "Rhetoric Theory" (Barthes, 1964). The results of the sentences show that students' perceptions tend towards collectivist and mixed motivational domains, such as universalism and achievement, coinciding with those shown by the results of the drawings. Other values related to inclusion emerge in turn, as self-direction and benevolence.
\end{abstract}

Keywords: inclusion, perception, disability, physical education, inclusive sport. 
${ }^{1}$ Correspondencia: Carmen Ocete Calvo. Centro de Estudios sobre el Deporte Inclusivo. Facultad de Ciencias de la Actividad Física y Del Deporte-INEF. Universidad Politécnica de Madrid. Calle Martín Fierro, nº 7, $3^{\mathrm{a}}$ planta, 28040 Madrid / Teléfonos: 913364140 /913364137 / carmina120@hotmail.com

\section{La inclusión de alumnos con discapacidad en Educación Física}

La trasformación educativa experimentada en los últimos años a favor de generar entornos inclusivos, donde los alumnos con discapacidad aprenden junto con el resto de sus compañeros (Campos, Ferreira, y Block, 2013; Block, 2007), ha aumentado las investigaciones sobre los posibles efectos sobre el alumnado sin discapacidad desde distintas perspectivas (McKay, Block, y Yeon, 2015; Perez-Tejero y Ocete, 2016). El área de Educación Física (EF) se muestra idónea para el trabajo en valores (Gutiérrez, 1995), facilitándolo a través de planteamientos metodológicos inclusivos (Ocete, Pérez, y Coterón, 2015).

La actitud, identificada como uno de los principales problemas de la educación inclusiva (Nowicki y Sandieson, 2002), es un factor determinante para la consecución del éxito (Conaster, Block, y Gansneder, 2002; Folsom-Meek y Rizzo, 2002), por este motivo, muchos autores se han focalizado en medir la actitud de alumnos sin discapacidad hacia la inclusión de sus compañeros que sí la tienen (Block, 1995; Cordente-Mesas, González-Víllora, Block, y Contreras-Jordán, 2016; Duchane y French, 1998; Kalyva y Agaliotis, 2013; Özer et al., 2012; Reina, López, Jiménez, García-Calvo, y Hutzler, 2011; Slininger, Sherrill, y Jankowski, 2000; Van Biesen, Busciglio, y Vanlandewijck, 2006; Verderber, Rizzo, y Sherrill, 2003).

Con el fin de esclarecer las mejores condiciones posibles para una práctica inclusiva en $\mathrm{EF}$, se han de evaluar tanto el diseño e implementación de las intervenciones como su efecto sobre el alumnado (Ocete, 2016). De esta forma, se pueden establecer patrones y estrategias óptimas para promover interacciones sociales positivas, donde el alumnado con discapacidad se sienta aceptado, motivado y en las que participe activamente (Spencer-Cavaliere y Watkinson, 2010), formando parte de las clases donde diariamente se producen aprendizajes efectivos a través de estas interacciones con el resto de sus compañeros (Hendrickson, Shokoohi-Yekta, Hamre-Nietupski, y Gable, 1996).

Un ejemplo de programa de intervención educativo es "Deporte Inclusivo en la Escuela" (Ocete y cols., 2015), con los objetivos de promover la práctica deportiva inclusiva en los centros educativos, dar a conocer los diferentes deportes paralímpicos mediante una metodología con carácter inclusivo y concienciar sobre la situación de las personas con discapacidad en la práctica deportiva (Pérez-Tejero, Barba, García-Abadía, Ocete, y Coterón, 2013). Está compuesto por una serie de recursos didácticos, además de una ponencia de un deportista paralímpico y un evento deportivo inclusivo: "Día del Deporte Inclusivo en la Escuela" (Ocete et al., 2015).

Este estudio está enmarcado en una de las actividades llevadas a cabo en el evento, por lo que describimos sus características principales con el fin de contextualizarlo. El evento tuvo lugar en las instalaciones de la Facultad de Ciencias de la Actividad Física y el Deporte-INEF y del Consejo Superior de Deportes (26 de abril de 2013). Fue planteado con una metodología inclusiva dada la participación conjunta entre alumnos con y sin discapacidad, donde los alumnos de secundaria y bachillerato practicaron diferentes deportes adaptados y paralímpicos 
(baloncesto y rugby en silla de ruedas, boccia, atletismo ciegos, fútbol 5 para ciegos, fútbol 7 para parálisis cerebral), así como una actividad de reflexión sobre la inclusión de compañeros con discapacidad en las sesiones de EF (Gavilanes, 2013), objeto de análisis en este trabajo.

La Teoría de la Retórica visual (Barthes, 1964), utilizada en numerosos estudios (Cao, 1998; Barthes, 2001; Menéndez Pidal, 2010), postula que todo dibujo constituye un texto visual en el que el mensaje se lee y se comprende como cualquier otro, verbal o no verbal, porque dibujar significa objetivar una idea, visualizarla en una forma y definirla gráficamente sobre una superficie. El dibujo es uno de los instrumentos con el que podemos indagar y comprender la realidad en la que vivimos (Lazotti, 1994), además de una exteriorización de un esquema mental que ha sido anteriormente interiorizado (Pérez y Suárez, 2004). La retórica visual es un modo de análisis que nos puede permitir articular la forma y el contenido de las imágenes que vemos (Cao, 1998).

Por este motivo, el objetivo de este estudio fue conocer la percepción de los alumnos sin discapacidad sobre la inclusión de compañeros que sí la tienen en las clases de EF, a través del análisis de los dibujos y frases expresadas durante la actividad de reflexión en el evento deportivo inclusivo indicado.

\section{MÉTODO}

\section{Muestra}

La muestra de este estudio corresponde a 700 alumnos de 14 centros educativos de Educación Secundaria Obligatoria y Bachillerato de la Comunidad de Madrid (9 centros ordinarios, 4 de atención preferente de motóricos y 1 de atención preferente de discapacidad auditiva) que participaron durante el curso 2012-13 en el programa DIE. Los sujetos, 50 alumnos por centro, fueron seleccionados para participar en el evento bajo criterio de sus profesores, teniendo prioridad de participación aquellas clases que tenían alumnos con discapacidad. Dado que los dibujos y frases fueron registradas de manera anónima, no podemos describir las variables de género, edad y tipo de centro educativo, considerándose una limitación del presente estudio.

\section{Instrumentos}

Se utilizó los seis murales de papel en los que los alumnos expresaron sus opiniones sobre la discapacidad, la inclusión y el deporte inclusivo mediante frases, palabras y/o dibujos.

\section{Procedimiento}

Comenzó con la organización de la información dependiendo si era frase o imagen. En el primer caso, se transcribió cada una de las frases y palabras escritas en los murales a un

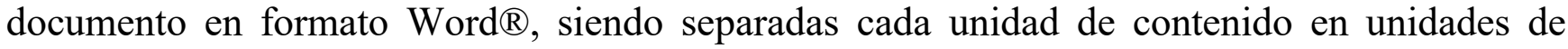
registro, obteniendo un total 178 frases y 22 palabras. Los dibujos presentes en los murales fueron fotografiados, digitalizados y archivados.

Una vez recogida toda la información, se siguieron una serie de fases para el análisis cualitativo de los textos (Heinemann, 2003). En primer lugar se inició una lectura intensiva flotante del documento para crear un esquema general del contenido y en segundo lugar, se diseñó una propuesta de categorías identificables en el texto. Tras una codificación inductiva o 
abierta (Strauss y Corbin, 2002), se procedió al análisis de las frases y palabras, con el fin de desarrollar un sistema de categorías cumpliendo las condiciones de homogeneidad, exhaustividad, exclusión mutua, objetividad, pertinencia y productividad (modificado de Berelson, 1952 y Bardin, 1986). Para este proceso se utilizó el programa NVIVO v10.

Tras comprobar la tendencia en las frases a expresar valores, el sistema de categorías se fundamentó en la "Teoría de la estructura y el contenido universal de los valores humanos" (Schwartz, 1992), que tras realizar un estudio en 60 culturas diferentes (Schwartz y Bilsky, 1987, 1990), postuló diez dominios motivacionales compuestos por 57 valores universales agrupados en cuatro tipologías según el tipo de objetivo motivacional que persiguen (transcendencia, promoción personal, apertura al cambio y conservación).

Tabla 1. Dominios motivacionales pertenecientes a la "Teoría de la estructura y el contenido universal de los valores humanos" (Schwartz, 1992).

\begin{tabular}{|c|c|}
\hline Dominio motivacional & Valores \\
\hline Autopromoción & $\begin{array}{l}\text { Poder: status social y prestigio, control o dominio sobre personas y recursos (poder social, } \\
\text { riqueza, autoridad). } \\
\text { Logro: éxito personal demostrando competencia de acuerdo a los estándares sociales } \\
\text { (exitoso, capaz, ambicioso, influyente). }\end{array}$ \\
\hline Apertura al cambio & $\begin{array}{l}\text { Hedonismo: Placer o gratificación sensual hacia uno mismo (placer, disfrutar de la vida). } \\
\text { Estimulación: Excitación, novedad y desafíos en la vida (arriesgado, vida variada, vida } \\
\text { excitante). } \\
\text { Auto-dirección: Independencia de pensamiento y acción -escoger, crear, explorar } \\
\text { (creatividad, libertad, curioso, independiente, escogiendo sus propios objetivos). }\end{array}$ \\
\hline Autotrascendencia & $\begin{array}{l}\text { Universalismo: entendimiento, apreciación, tolerancia y protección por el bienestar de } \\
\text { todas las personas y por la naturaleza (justicia social, amplitud mental, mundo en paz, } \\
\text { cordura, un mundo de belleza, unidad con la naturaleza, proteger el ambiente, igualdad). } \\
\text { Benevolencia: Preservación y realce del bienestar de las personas con los cuales uno está } \\
\text { en contacto personal frecuente (dispuesto a ayudar, perdonar, honesto, amistad verdadera, } \\
\text { lealtad). }\end{array}$ \\
\hline Conservación & $\begin{array}{l}\text { Tradición: respeto, compromiso y aceptación de las costumbres e ideas que la propia } \\
\text { cultura o religión impone a la persona (aceptar mi parte en la vida, devoto, respeto por la } \\
\text { tradición, humildad, moderación). } \\
\text { Conformidad: Restricción de acciones, inclinaciones e impulsos que pueden perjudicar a } \\
\text { otras personas } \\
\text { Seguridad: seguridad, armonía y estabilidad social en las distintas relaciones y consigo } \\
\text { mismo. }\end{array}$ \\
\hline
\end{tabular}

Para su categorización, se creó una ficha basada en la propuesta por Ocete (2014), donde se definían cada uno de los dominios, valores y algunos ejemplos para cada uno de ellos. Se llevó a cabo un nuevo análisis del contenido para resolver dudas y hacer las modificaciones que se considerasen pertinentes según criterio experto y presentar la propuesta de sistema de categorías definitivo sobre el cual categorizar las respuestas del alumnado.

En cuanto al análisis de los dibujos, se realizó un estudio exhaustivo de cada una de ellas basándonos en la "Teoría de la retórica visual" (Barthes, 1964), donde cabe destacar que la repetición de una imagen o un elemento puede significar la expresión enfática de la multiplicidad. Por otro lado, las figuras de supresión de determinados elementos nos pueden mostrar cuales son los elementos esenciales. También es muy utilizada en la retórica visual, la técnica de la personificación de objetos no humanos mediante las imágenes (Cao, 1998), por lo 
que el análisis retórico de los dibujos nos permitió entender las percepciones de los alumnos participantes. Tras el análisis, se observó una tendencia a manifestar valores, por lo que se creó una ficha de categorización basada en la propuesta por Ocete (2014), donde los dibujos se organizaban según el objetivo motivacional que perseguían (transcendencia, promoción personal, apertura al cambio y conservación), fundamentando en la "Teoría de la estructura y el contenido universal de los valores humanos" (Schwartz, 1992).

En un último análisis realizado por grupo de expertos en metodología se decidió que algunos dibujos podían ser categorizados en varios dominios motivacionales a la vez. En ambos análisis (frases y dibujos) se asignó un código de categorización para identificar las unidades de registro, identificando el dominio motivacional y el número de la frase o imagen (por ejemplo, UDı21: unidad de registro de Logro, número 21).

\section{RESULTADOS}

\section{Frases y palabras}

El número total de unidades de registro obtenidas es de 296, categorizadas en los 10 dominios motivacionales (Schwartz, 1992).

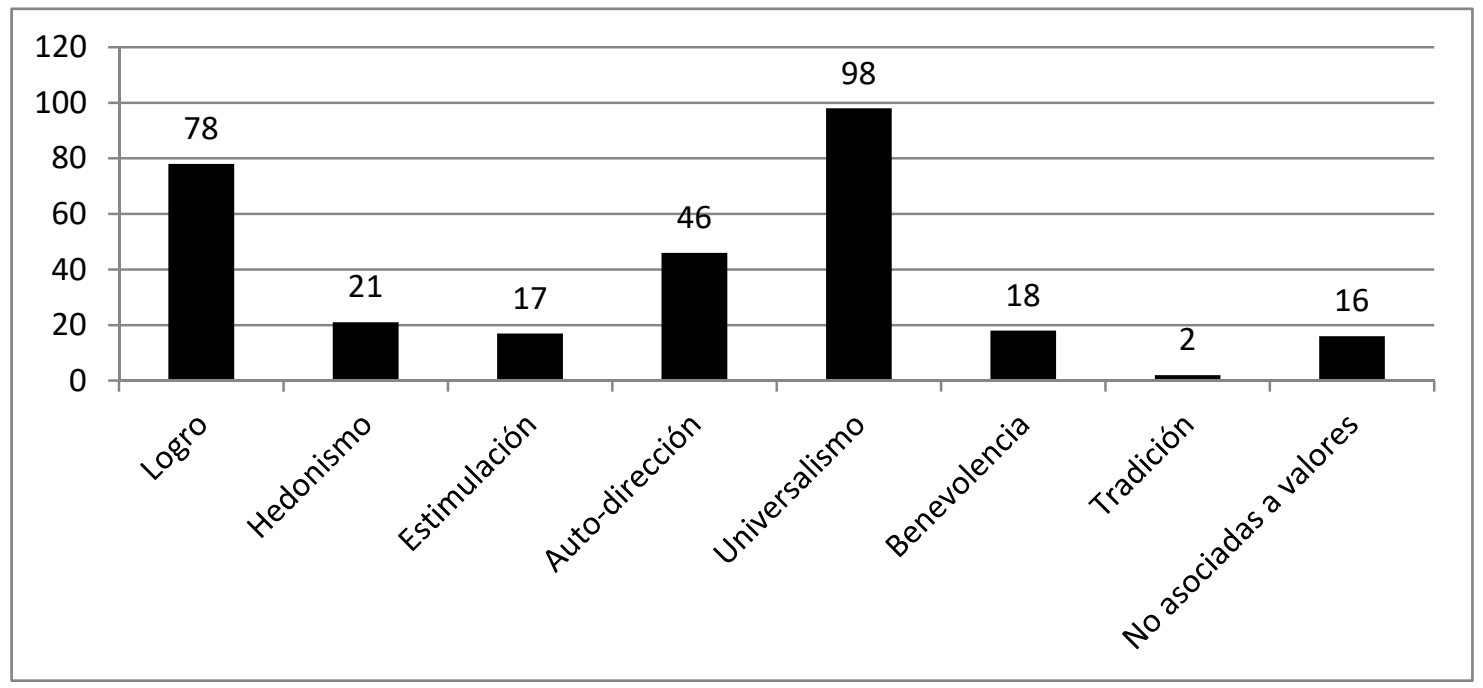

Figura 1. Frecuencias de los dominios motivacionales en el análisis de las frases y palabras.

En la Figura 1 se observan los dominios "universalismo" y "logro", como los más destacados, seguidos de "autodirección", "hedonismo", "benevolencia" y "estimulación". Por último, y con un porcentaje inferior y no significativo se da el dominio motivacional de la "tradición". En este análisis, no se encuentran frases relacionadas con los dominios de "poder", "seguridad" y "conformidad", por lo que el sistema de categorías presentado muestra tan solo 7 de los 10 dominios motivacionales de la teoría. 
Tabla 2. Algunos ejemplos de dominios motivacionales categorizados

\begin{tabular}{|c|c|c|}
\hline Dominio motivacional & Ejemplo & Código \\
\hline Logro & $\begin{array}{l}\text { "Es una forma de darnos cuenta del esfuerzo que hacen las personas } \\
\text { discapacitadas y aprender de ello" }\end{array}$ & $\left(\mathrm{UD}_{\mathrm{L}} 21\right)$ \\
\hline Hedonismo & "Pasando un buen momento" & $\left(\mathrm{UD}_{\mathrm{H}} 4\right)$ \\
\hline Estimulación & "¡Todos podemos, estamos en la misma sociedad!" & $\left(\mathrm{UD}_{\mathrm{E}} 2\right)$ \\
\hline Autodirección & $\begin{array}{l}\text { "Podemos aprender mucho de las personas con discapacidad, al igual } \\
\text { que ellos pueden aprender de nosotros" }\end{array}$ & $\left(\mathrm{UD}_{\mathrm{A}} 2\right)$ \\
\hline Universalismo & "Con o sin discapacidad todos somos iguales" & $\left(\mathrm{UD}_{\mathrm{U}} 3\right)$ \\
\hline Benevolencia & $\begin{array}{l}\text { "Cuando se está en una silla de ruedas me sentí mal por no mover las } \\
\text { piernas y mucho peor por no poder andar." }\end{array}$ & $\left(\mathrm{UD}_{\mathrm{B}} 10\right)$ \\
\hline Tradición & $\begin{array}{l}\text { "Es muy difícil hacer que la sociedad acepte a los discapacitados ya } \\
\text { que siempre se tiende a rechazar a los diferentes" }\end{array}$ & $\left(\mathrm{UD}_{\mathrm{T}} 1\right)$ \\
\hline
\end{tabular}

\section{Dibujos}

El número total obtenidos y analizados es de 50, aunque alguna de ellas fue categorizada en varios dominios, por lo que muestran 61 dominios motivacionales. Como se puede observar en la Figura 2 solamente se han dado cinco dominios motivacionales:

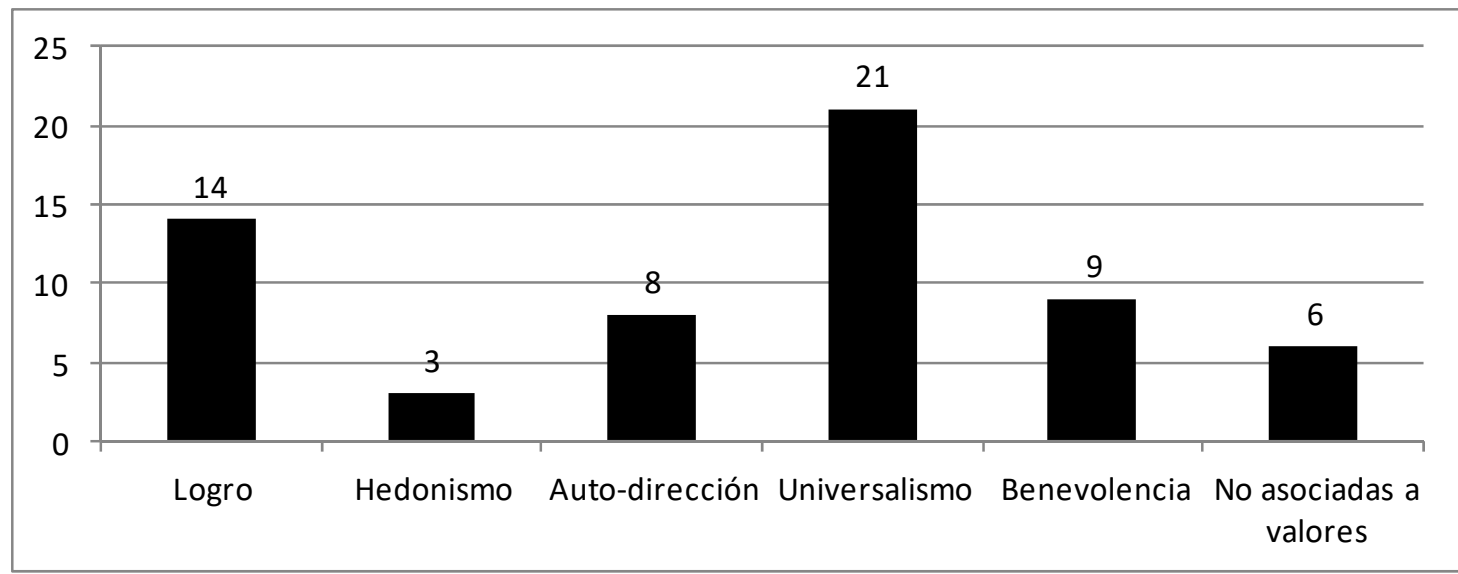

Figura 2. Frecuencias de los dominios motivacionales en el análisis de los dibujos

A continuación se muestran algunos ejemplos de dibujos con sus dominios motivacionales correspondientes:

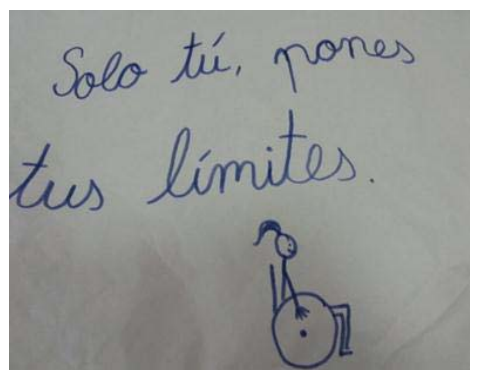

Figura 3. Dibujo categorizado en el dominio motivacional de logro 


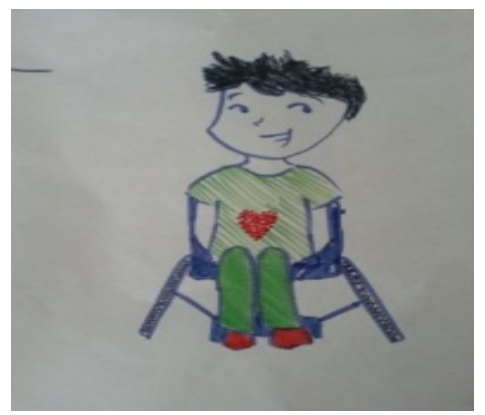

Figura 4. Dibujo categorizado en el dominio motivacional de hedonismo

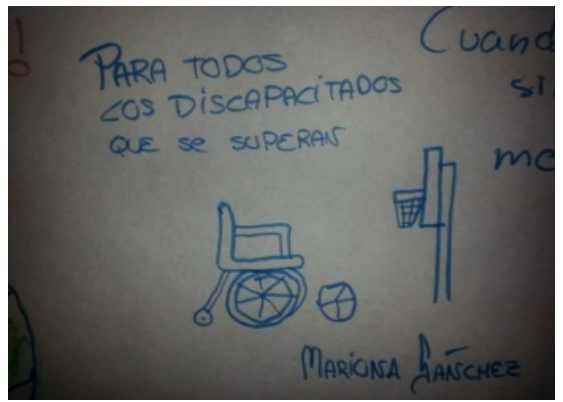

Figura 5. Dibujo categorizado en el dominio motivacional de autodirección

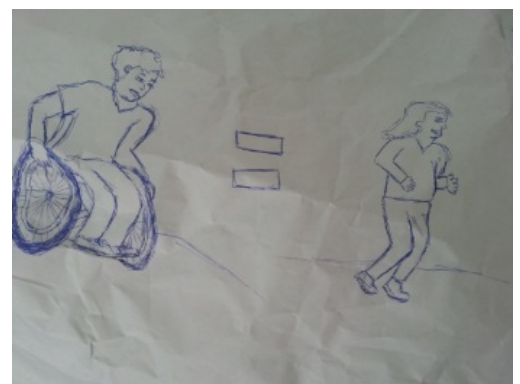

Figura 6. Dibujo categorizado en el dominio motivacional de universalismo

\section{DISCUSIÓN}

Dada la originalidad del trabajo, hace difícil su comparación en la bibliografía con otros estudios, máxime si la comparación se contextualiza en el área de EF. Por tanto, la aplicación de la Teoría de la estructura y el contenido universal de los valores humanos (Schwartz, 1992) para el análisis de las creencias del alumnado hacia la inclusión de compañeros con discapacidad en EF, es una de las aportaciones de este trabajo.

Dado que las creencias conductuales manifiestan la evaluación de un sujeto acerca de algo concreto, según la TCP (Ajzen, 1991), el análisis de estas creencias arroja en nuestro caso los intereses y metas motivacionales de los alumnos hacia la inclusión de compañeros con discapacidad, reflejando sus objetivos y los valores personales (Ocete, 2016). Los valores emergentes en este análisis, ponen de manifiesto la importancia de conocer la percepción de los alumnos en este tema, con el fin de diseñar intervenciones futuras de calidad que se ajusten al perfil del alumnado. Otra de las aportaciones de este trabajo, es el uso de la Teoría de la Retórica (Barthes, 1964) aplicada en para el análisis de los dibujos en las sesiones de EF. 


\section{Frases y palabras}

En relación a las frases y las palabras analizadas, el valor más destacado es el universalismo, perteneciente a la dimensión de autotrascendencia con naturaleza colectivista, donde se identifica la inclusión de compañeros con discapacidad con un mundo armonioso y un "todo", mostrando interés por el bienestar de los demás, por encima de los suyos propios (Ocete, 2016). Schwartz (2007) postuló que los valores pertenecientes a este dominio predicen la voluntad de aceptar a minorías en peligro de exclusión social, como puede ser por motivos de raza o discapacidad en este caso, enfatizando en valores como la aceptación, el aprecio y la preocupación por el bienestar de todos los demás. Coincidiendo con estos resultados, encontramos el estudio llevado a cabo por Vecchione, Caprara, Schoen, González y Schwartz (2012) sobre una muestra de 1.569 sujetos procedentes de España, Alemania e Italia. Como resultado obtuvieron la predominancia de este valor sobre el resto, ayudando en la predicción de la percepción de las consecuencias de la inmigración. En esta misma línea están los resultados encontrados por Hendrickson, Shokoohi-Yekta, Hamre-Nietupski y Gable (1996) tras examinar los valores predominantes de alumnos sin discapacidad acerca de formar amistades con sus compañeros que sí la tenían.

Los resultados muestran además los dominios motivacionales de logro y autodirección, mostrando la esencia de los intereses del alumnado. En el caso del primero (dimensión autopromoción), pone énfasis en la superioridad social y la estima, en sentirse exitoso, mientras que el segundo (dimensión apertura al cambio), muestra el interés por la novedad, así como la intención de tomar sus propia decisiones. En esta línea, coincidimos con Döring, Blauensteiner, Aryus, Drögekamp y Bilsky, (2010), siendo explicado como la manifestación por ser mejor persona, superarse para conseguir metas o para hacer a sus padres felices en su caso. Ocete (2016) defiende que se puede inferir que los alumnos ven las situaciones que se producen cuando están en contacto con compañeros con discapacidad como oportunidades de superarse a sí mismos y de mejorar como personas, con el objetivo prioritario de sentirse bien con ellos mismos. Respecto al hedonismo, los alumnos reflejaban disfrutar a través de la práctica inclusiva; mientras que con la benevolencia los alumnos muestran su disposición a ayudar. Coincidiendo con estos resultados Cohen (2010), en su caso con profesores israelíes, lo relaciona con la solidaridad, la cooperación, la confianza mutua y el apoyo, valores que pueden ser favorecedores de la inclusión.

Por último, el tipo motivacional menos predominante, entre los que se han dado, ha sido la tradición, relacionado con la imposibilidad de la igualdad entre personas con y sin discapacidad. El hecho de no encontrar frases negativas, puede venir determinado por haber recibido la intervención del programa y tener un posible efecto sobre el alumnado, convirtiéndose en un sesgo de los resultados y una limitación del estudio.

En contraste con estos resultados, existe una ausencia de dominios motivacionales como la conservación y la seguridad relacionados con valores opuestos a la aceptación, ya que representan la protección personal y social por el mantenimiento de las normas, y la preservación de las tradiciones de la sociedad en la que viven, como muestran en sus estudios Schwartz $(2006,2007)$ y Myyry y Helkama (2001), donde se mostraba una correlación negativa con la empatía, entendida como una habilidad cognitiva y emocional del individuo, en la que es capaz de ponerse en la situación emocional de otro. 


\section{Dibujos}

Siguiendo a Pérez y Suárez (2007), entendemos que los dibujos de los alumnos son imágenes mentales que han interiorizado llegando a alcanzar una de los objetivos del programa DIE, la sensibilización hacia la discapacidad. El análisis de los dibujos nos arroja resultados muy semejantes a las frases y las palabras. Coincidiendo como destacado el dominio motivacional de universalismo. Desde el análisis de la retórica, cuando en un dibujo se colocan juntas la persona sin discapacidad y con discapacidad (ambas figuras son iguales pero a una se le añaden la silla de ruedas, por ejemplo) se está mostrando la percepción de igualdad. En el caso del logro son ilustraciones que muestran la capacidad de los alumnos con discapacidad para desempeñar las mismas actividades deportivas que los alumnos sin discapacidad tanto de forma cooperativa como competitiva. El tercer dominio motivacional predominante fue la benevolencia, representada mediante dibujos que muestran la búsqueda del bienestar de las personas que tenemos a nuestro alrededor así como la disposición a ayudarlas. Los alumnos mostraban la unión a través de un objeto simbólico como es el balón, puesto que implica la participación conjunta y el contacto entre las personas que aparecen. Los dibujos categorizados en hedonismo muestran la felicidad de las personas con discapacidad al realizar actividad física de forma conjunta con el resto de alumnos, pudiendo deberse al efecto del impacto del programa en su percepción. En el análisis, se han encontrado 6 dibujos que no han podido ser asociados a valores, destacando entes similares a extraterrestres, lo que nos puede inducir a pensar que se trate de alumnos que no hayan tenido nunca contacto con personas con discapacidad o son dibujos sin relación con la actividad indicada.

Apoyándonos en la Teoría de la Retórica, dentro de las principales figuras de adjunción se debe destacar que la repetición de una imagen o un elemento puede significar la expresión enfática de la multiplicidad, en el caso de este trabajo, la silla de ruedas, imagen aceptada universalmente como símbolo de la accesibilidad, al igual que el bastón como elemento representativo de la discapacidad visual. Esto no significa que los alumnos rechacen o no conozcan más discapacidades sino que identifican la discapacidad con estos símbolos. A su vez, las figuras de supresión de determinados elementos nos pueden mostrar cuales son los elementos esenciales. Según la retórica, mediante la representación de un grupo se manifiesta que cada miembro del grupo es exactamente igual (aunque no sean físicamente similares) porque tienen una constante: que son deportistas y pertenecen al mismo equipo donde las características individuales no son una barrera. Tanto en las frases como en los dibujos, predominan valores relacionados con el éxito personal a través de conseguir el bienestar de los demás por encima del propio.

Una de las limitaciones encontrada en este trabajo fue que al ser anónimos los dibujos y frases, no se puede caracterizar la muestra, no pudiendo hacer una distinción por género, edad y tipo de centro escolarizado, siendo variables determinantes en el estudio de la percepción de la inclusión de alumnos con discapacidad. Por tanto, estas limitaciones ponen de manifiesto, la necesidad de futuros trabajos donde se consideren estas cuestiones. Además, sería aconsejable realizar una toma de datos previa a la participación de los alumnos en la actividad, con el fin de un análisis comparativo de los resultados. 


\section{CONCLUSIONES}

La percepción de los alumnos hacia la inclusión de compañeros con discapacidad refleja una tendencia hacia valores relacionados con el éxito personal a través de conseguir el bienestar de los demás, la tolerancia y la igualdad, valorando de forma positiva la inclusión de compañeros con discapacidad en las clases de EF.

\section{AGRADECIMIENTOS}

Queremos agradecer el apoyo recibido por el Consejo Superior de Deportes, el Comité Paralímpico Español, al INEF-UPM y a los profesores de EF así como a sus alumnos de secundaria participantes

\section{REFERENCIAS}

Ajzen, I. (1991). The Theory of Planned Behavior. Organizational behavior and human decision processes, 50, 179-211.

Bardin, L. (Ed.). (1986). El análisis de contenido. Madrid: Akal.

Barthes, R. (1964). Réthorique de l'image. Communications, (27), 40-51.

Barthes, R. (2001). La Torre Eiffel. Textos sobre la imagen. España: Paidós Comunicación.

Berelson, B. (Ed.). (1952). Content analysis in communication researches. Glencoe III: Free Press.

Block, M. (1995). Development and validation of the Children's Attitudes Toward Integrated Physical Education -- Revised (CAIPE-R) inventory. Adapted Physical Activity Quarterly, 12(1), 60-77.

Conaster, P., Block, M. E., y Gansneder, B. (2002). Aquatic instructors' beliefs toward inclusion: the theory of planned behavior. Adapted physical activity quarterly, 19(2), 172-187.

Cordente-Mesas, D., González-Víllora, S., Block, M. E., y Contreras-Jordán, O. (2016). Structure, validity and reliability of the Children's Attitudes Towards Integrated Physical Education-Spanish version (CAIPE-SP). European Journal of Adapted Physical Activity, 9(2), 3-12.

DöRing, A., Blauensteiner, A., Aryus, K., DröGekamp, L., y Bilsky, W. (2010). Assessing Values at an Early Age: The Picture-Based Value Survey for Children (PBVS-C). Journal of Personality Assessment, 92(5), 439-448.

Duchane, K., y French, R. (1998). Attitudes and Grading Practices of Secondary Physical Educators in Regular Education Settings. Adapted Physical Activity Quarterly, 15(4), 370-380.

Folsom-Meek, S., y Rizzo, T. (2002). Validating the Physical Educators' Attitude Toward Teaching Individuals With Disabilities III (PEATID III) Survey for Future Professionals. Adapted Physical Activity Quarterly, 19(2), 141.

Gutiérrez, M. (Ed.). (1995). Valores sociales y deporte. Editorial Gymnos, S.L.

Heinemann, K. (Ed.). (2003). Introducción a la metodología de la investigación empírica en las ciencias del deporte. Barcelona: Paidotribo. 
Hendrickson, J., Shokoohi-Yekta, M., Hamre-Nietupski, S., y Gable, R. (1996). Middle and high school students' perceptions on being friends with peers with severe disabilities. Exceptional Children, 63(1), 19-19.

Kalyva, E., y Agaliotis, I. (2013). Can Contact Affect Greek Children's Understanding of and Attitudes towards Peers with Physical Disabilities? European Journal of Special Needs Education, 24(2), 213-220.

McKay, C., Block, M. E., y Yeon, J. (2015). The Impact of Paralympic School Day on Student Attitudes Toward Inclusion in Physical Education. Adapted Physical Activity Quarterly, 32, $331-348$.

Myyry, L., y Helkama, K. (2001). University students' value priorities and emotional empathy. Educational Psychology, 21, 25-40.

Nowicki, E., y Sandieson, R. (2002). A Meta-Analysis of School-Age Children's Attitudes towards Persons with Physical or Intellectual Disabilities. International Journal of Disability, Development and Education, 49(3), 243-265.

Ocete, C., Pérez, J., y Coterón, J. (2015). Propuesta de un programa de intervención educativa para facilitar la inclusión de alumnos con discapacidad en Educación Física. Retos. Nuevas tendencias en Educación Física, Deporte y Recreación(27), 140-145.

Özer, D., Baran, F., Aktop, A., Nalbant, S., Ağlamış, E., y Hutzler, Y. (2012). Effects of a Special Olympics Unified Sports soccer program on psycho-social attributes of youth with and without intellectual disability. Research in Developmental Disabilities, 33(1), 229-239.

Pérez-Tejero, J., Barba, M., García-Abadía, L., Ocete, C., y Coterón, J. (2013). Deporte Inclusivo en la Escuela. Madrid: Universidad Politécnica de Madrid.

Perez-Tejero, J., y Ocete, C. (2016). Influencia de la práctica deportiva inclusiva en las personas con discapacidad. . En J. Segura (Ed.), Psicología aplicada al deporte adaptado (pp. 169-179): Editorial UOC.

Reina, R., López, V., Jiménez, M., García-Calvo, T., y Hutzler, Y. (2011). Effects of awareness interventions on children's attitudes toward peers with a visual impairment. International Journal of Rehabilitation Research, 34 (3), 243-248.

Schwartz, S. (1992). Universals in the content and Structure of Values: Theoretical Advances and Empirical Tests in 20 Countries Advances in Experimental. Social Psychology of Education:An International Journal, 25, 1-65.

Schwartz, S. (2006). Basic human values and theory: measurement and applications. Revue française de sociologie, 47(4), 929-968.

Schwartz, S. (2007). Value orientations: Measurement, antecedents and consequences across nations. En C. R. Jowell, R. Fitzgerald, \& G. Eva (Ed.), Measuring attitudes crossnationally: Lessons from the European Social Survey (pp. 169-203). London: Sage.

Schwartz, S., y Bilsky, W. (1987). Toward a universal psychological structure of human values. Journal of Personality and Social Psychology, 53, 550-562.

Schwartz, S., y Bilsky, W. (1990). Toward a theory of the universal content and structure of values: extensions and cross-cultural replications. Journal of Personality and Social Psychology, 58, 878-891. 
Slininger, D., Sherrill, C., y Jankowski, C. (2000). Children's Attitudes Toward Peers With Severe Disabilities: Revisiting Contact Theory. Adapted Physical Activity Quarterly, 17(2), 176-196.

Spencer-Cavaliere, N., y Watkinson, E. (2010). Inclusion Understood From the Perspectives of Children With Disability. Adapted Physical Activity Quarterly, 27(4), 275-293.

Strauss, A. L., y Corbin, J. (Eds.). (2002). Bases de la investigación cualitativa: técnicas y procedimientos para desarrollar la teoría fundamentada. Medellín, Colombia: Universidad de Antioquía.

Van Biesen, D., Busciglio, A., y Vanlandewijck, Y. (2006). Attitudes towards inclusion of children with disabilities: the effect of the implementation of "A Paralympic School Day" on Flemish elementary children. 8th European Conference of Adapted Physical Activity Revisado el 26 octubre de 2011, recuperado de http://www.eufapa.upol.cz/index.php/eucapa/proceedings/eucapa-2006.html

Vecchione, M., Caprara, G., Schoen, H., Gonzàlez, J. L., y Schwartz, S. (2012). The role of personal values and basic traits in perceptions of the consequences of immigration: A three-nation study. British Journal of Psychology, 103(3), 359-377.

Verderber, J., Rizzo, T., y Sherrill, C. (2003). Assessing student intention to participate in inclusive physical education. Adapted Physical Activity Quarterly, 20(1), 26-45. 